\title{
Vom schleichenden Ende der Nahversorgung
}

\author{
Während die meisten Städte in Deutschland \\ über ein attraktives Versorgungsangebot \\ verfügen, muss der Verbraucher in ländlichen \\ Räumen weite Wege zurücklegen, um tägliche \\ Besorgungen erledigen zu können. Die lokale \\ Versorgung mit Waren des täglichen Bedarfs \\ ist die Ausnahme und nicht die Regel. \\ Von Christian Kuhlicke und Ulrich Petschow
}

W ird die Gleichwertigkeit der Lebensverhältnisse infrage gestellt, folgt der Aufruhr zumeist sofort. Eine mögliche Einschränkung des Rechts auf Gleichwertigkeit hätte nicht nur praktische Auswirkungen für den Einzelnen, sie nagt auch am Selbstverständnis einer Nation. Faktisch schreitet die Aufhebung der Gleichwertigkeit auch in einem Bereich voran, der von Politik und Öffentlichkeit kaum ins Auge gefasst wird: der Nahversorgung. Eine Primärerhebung des Instituts für ökologische Wirtschaftsforschung (IÖW) zur Versorgungssituation in ländlichen Landkreisen zeigt, dass im Gegensatz zur Großstadt die Entfernung zwischen Heim und Verkaufsstellen in ländlichen Gebieten steigen. Das stellt gerade weniger mobile Bevölkerungsgruppen wie ältere und behinderte Menschen sowie Bürger ohne Auto vor Herausforderungen. Gerade sie sind auf lokale Versorgung angewiesen.

Fasst man die zukünftige Bevölkerungsentwicklung in den ländlichen Räumen ins Auge, zeichnet sich hier ein Teufelskreis von schrumpfender Bevölkerung und abnehmender Kaufkraft, Rückzug des Handels aus der Fläche und Verringerung der individuellen sowie öffentlichen Mobilität ab. Diesen zu durchbrechen wird nicht allein im Rahmen gängiger marktlicher Verteilungsmuster möglich sein. Daher wird es Zeit, eine vor 20 Jahren beginnende Entwicklung verstärkt ins öffentliche Interesse zu rücken: das Ende der Nahversorgung im ländlichen Raum. Nachfolgend werden zentrale Ergebnisse der Studie des IÖW anhand der ländlichen Landkreise Daun und BitburgPrüm vorgestellt. Wie viele Verkaufsstellen in diesen Kreisen existieren und wie sie räumlich verteilt sind, bilden die zentralen Fragen der Untersuchung.

\section{Die Angebotsseite}

Gerade in den letzten zwei Jahrzehnten unterliegt der Lebensmitteleinzelhandel einem tief greifenden Strukturwandel.
Die Konzentration auf der Unternehmensebene und die Konzentration der Standorte nehmen ebenso zu wie die Zahl der filialisierten gegenüber den selbstständig geführten Lebensmitteleinzelhändlern. So hatten im Jahre 2000 die Top 5 Unternehmen im deutschen Lebensmitteleinzelhandel einen Marktanteil von 62,3 Prozent ( $\mathrm{M}+\mathrm{M}$ Eurodata 2000) (1). In Bezug auf die Einkaufsstätten stellen die drei nach Anzahl der Geschäfte größten Gruppen Edeka, Rewe und Spar knapp 50 Prozent der Einkaufsstätten. Sie haben demzufolge große Bedeutung für die Aufrechterhaltung der Nahversorgung.

Aber auch die expandierenden Discounter übernehmen mit der Ausdehnung ihres Handelsnetzes zusehends eine Nahversorgungsfunktion. Die Discounter haben in diesem Zusammenhang allerdings eine ambivalente Wirkung, da sie sowohl ein relativ begrenztes Sortiment anbieten als auch aufgrund ihrer Niedrigpreisstrategien eine Bedrohung für den traditionellen Einzelhandel sind und damit zu einer weiteren Ausdünnung der Vielfalt des Nahversorgungsangebots beitragen können.

Es sind vor allem die kleineren Geschäfte unter 800 Quadratmeter (qm), die noch immer den Großteil der Verkaufsstellen darstellen und die wesentlichen Träger der Nahversorgung sind. Allerdings machen die kleineren Geschäfte nur rund 20 Prozent des Umsatzes aller Einzelhändler aus (BVL 2004) - Tendenz abnehmend.

Betrachtet man die Umsätze nach Betriebsformen, so verzeichnen vor allem die Discounter steigende Umsätze. Diese haben sich zwischen 1991 und 2004 fast verdoppelt, während sie bei den Supermärkten und übrigen Lebensmittelgeschäften abgenommen haben (EHI 2004). Prognosen gehen davon aus, dass sich dieser Prozess bis zum Jahr 2010 noch deutlich verstärken wird. So wird vorhergesagt, dass im Jahr 2010 die Top 5 mit einem Marktanteil von 81,6 Prozent den Lebensmitteleinzelhandel weitgehend dominieren werden $(\mathrm{M}+\mathrm{M}$ Eurodata 2000).

Die Folge ist, dass sowohl die Konsumenten als auch die vorgelagerte Lebensmittelwirtschaft in stärkere Abhängigkeit von immer weniger Anbietern geraten. Die Folgen für den ländlichen Raum könnten hierbei besonders nachhaltig ausfallen. Es ist zu erwarten, dass die Top 5 in ihrer Standortwahl vermutlich nicht alle Regionen gleichmäßig berücksichtigen werden. Bevorzugt werden dürften diejenigen Gebiete, in denen die Kaufkraft hoch ist, benachteiligt werden die Regionen, die durch eine geringe Bevölkerungsdichte und eine geringe Kaufkraft charakterisiert sind, also vor allem die dünn besiedelten und schrumpfenden ländlichen Räume. 


\section{Die Nachfrageseite}

Die zwei für den Handel wichtigsten Veränderungen im Verhalten des Verbrauchers sind seine deutlich höhere Mobilität und sein gesteigertes Preisbewusstsein. Lag der Anteil der für Nahrungs- und Genussmittel aufgewendeten Beträge 1960 noch bei rund 9,5 Prozent des Bruttosozialprodukts, sind es heute rund 5,5 Prozent (EHI 2004). Es ist davon auszugehen, dass diese Entwicklung weiter anhält. So geht der Hauptverband des deutschen Einzelhandels davon aus, dass im Jahre 2020 nur noch rund 23 Prozent des privaten Konsumaufkommens für den Einzelhandel ausgegeben werden. Zum Vergleich, 1995 waren es noch rund 35 Prozent (IKB 2003).

Diese Entwicklung ist vor dem Hintergrund eines sich verändernden Konsumentenverhaltens zu sehen. Legte der durchschnittliche Konsument in fordistischen Zeiten ein eher einheitliches, relativ klar stratifiziertes Konsummuster an den Tag, scheint das post-fordistische Muster deutlich differenzierter ausgeprägt zu sein.

Abbildung 1: Kaufstruktur - gestern (links) sowie heute und morgen (rechts)

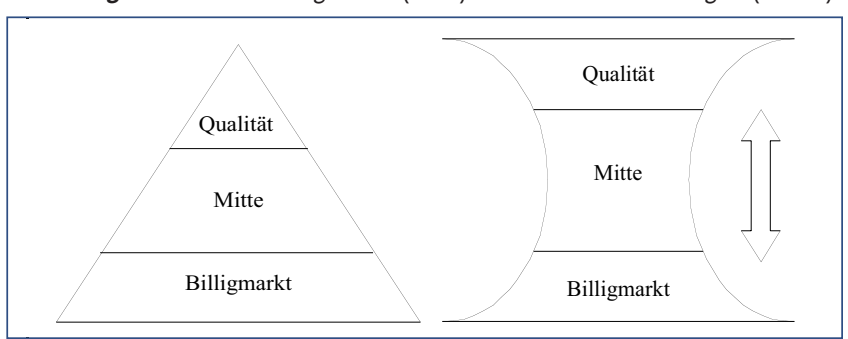

Quelle: Eigene Darstellung

Die linke Figur gibt das fordistische Konsummuster wieder. Hier herrscht auf relativ homogenen Märkten breite Nachfrage nach günstigen Produkten. An der Spitze der Pyramide existiert ein kleines Segment der Nachfrage nach Qualitätsprodukten. Die rechte Figur entspricht dem post-fordistischen Konsummuster. Hier nimmt einerseits der Preiskauf (Billigmarkt) einen starken Aufschwung. Andererseits erfolgt eine Ausdifferenzierung und Verbreiterung der Nachfrage nach Qualitäts- und Luxusprodukten.

In beiden Fällen sind die traditionellen Anbieter der Nahversorgung im Nachteil. Das Billigmarktsegment erfordert die Erschließung von Größenvorteilen, was kleinen Anbietern in der Regel verwehrt ist. Auch das ausdifferenzierte Qualitätssegment können kleinere Anbieter im ländlichen Raum nicht bereithalten. Vor dem Hintergrund einer zunehmend mobilen Bevölkerung in Verbindung mit ausdifferenzierten Nachfragestrukturen befinden sich die Nahversorger in einer wirtschaftlich ungünstigen Situation, da die Kaufkraft der weniger mobilen Teile der Bevölkerung für einen rentablen Betrieb nicht ausreicht. Es ist gerade diese Segmentierung der Nachfragemärkte, die eine wesentliche und größtenteils negative Bedeutung für die Nahversorgung hat. Zunehmend ausdifferenzierte Märkte können nicht oder nur noch begrenzt durch klas- sische Nahversorger bedient werden. Für den mobilen Konsumenten sind die damit verbundenen Konsequenzen kaum spürbar, für den nichtmobilen Verbraucher umso mehr. Nicht nur, dass beispielsweise ältere Menschen genau die kleinen, übersichtlichen und meist mit persönlicher Beratung verbundenen Läden bevorzugen, die allerdings zunehmend verdrängt werden (Federsl-Lieb 1992). Für sie gestaltet sich auch die Teilhabe an neuen Konsummustern und -möglichkeiten zunehmend schwierig. Wie sich die räumliche Konzentration der Verkaufsstellen derzeit darstellt, soll nachfolgend anhand von zwei ausgewählten Landkreisen exemplarisch gezeigt werden.

\section{Die Landkreise Daun und Bitburg-Prüm}

Die ländlichen Kreise Daun und Bitburg-Prüm liegen im Westen des Bundeslandes Rheinland-Pfalz. Daun hat eine Einwohnerdichte von $71 \mathrm{Ew} / \mathrm{qkm}$, bei einer Einwohnerzahl von 64.285 Personen. In Bitburg-Prüm wohnen 95.850 Personen, das entspricht $59 \mathrm{Ew} / \mathrm{qkm}$. Beide Landkrise erwartet eine leicht abnehmende Bevölkerung bei einer Zunahme der über 60-jährigen Einwohner bis 2020 (Bezugsjahr 1990) von rund 45 Prozent (Daun) beziehungsweise rund 35 Prozent (Bitburg-Prüm) (BBR 2003).

Um Aussagen über die Ausstattung der ausgesuchten Landkreise mit Lebensmitteleinzelhändlern machen zu können, wurden in einer Primärerhebung alle Einzelhändler der folgenden Sparten erhoben: Bäcker, Metzger und Fleischer, SB-Geschäfte unter 100 qm Geschäftsfläche, Supermärkte, Discounter sowie Warenhäuser. Datengrundlage waren die bei den jeweils zuständigen Industrie- und Handelskammern (IHK) und Gewerbeämter der Samtgemeinden geführten Händler.

In den 109 Gemeinden des Landkreises Daun wurden durch die Erhebung des IÖW insgesamt 97 Verkaufsstellen erfasst. Davon entfallen rund 30 Prozent auf größere Handelsgeschäfte wie Supermärkte und Discounter. 68 Prozent der Geschäfte sind Metzger, Bäcker oder SB-Geschäfte unter 100 qm (TanteEmma-Läden). Rund zwei Prozent konnten nicht zugeordnet werden. Die erfasste Verkaufsfläche der Lebensmitteleinzelhändler mit einer Verkaufsfläche von mehr als 100 qm beträgt $21.530 \mathrm{qm}$.

In Daun haben 74 der 109 Gemeinden keinerlei Versorgungseinrichtungen, was bedeutet, dass knapp 70 Prozent der Gemeinden ohne Verkaufstellen sind. In diesen 70 Prozent der Gemeinden wohnen 33 Prozent der Bevölkerung. Das Bild spitzt sich nochmals zu, wenn lediglich Einzelhandel mit einer Verkaufsfläche von mehr als 100 qm betrachtet wird. In Daun sind 88 Prozent der Gemeinden, in denen mehr als die Hälfte der Einwohner leben, ohne Lebensmitteleinzelhandel mit Verkaufsflächen von mehr als 100 qm.

Im Landkreis Bitburg-Prüm wurden in der Erhebung des IÖW 154 Lebensmitteleinzelhändler identifiziert, die sich zu 21 Prozent auf den großflächigen Lebensmitteleinzelhandel, zu 77 Prozent auf kleinere Verkaufsstellen und zu zwei Prozent auf Händler, die nicht zuzuordnen waren, verteilen. Die gesam- 
te Verkaufsfläche beläuft sich hier auf 43.750 qm. Auffällig ist im Landkreis Bitburg-Prüm der fast doppelt so hohe Anteil der Supermärkte gegenüber den Discountern. Hier leisten noch weitestgehend die Supermärkte einen wichtigen Beitrag zur Nahversorgung.

183 der 235 Gemeinden im Landkreis Bitburg-Prüm verfügen über keinerlei lokale Versorgungseinrichtungen. Das heißt, dass knapp 80 Prozent der Gemeinden ohne Verkaufstellen sind und fast 40 Prozent der Bevölkerung hier nicht an der Nahversorgung teilhaben können. Berücksichtigt man die Gemeinden, die mit einer Verkaufsstelle von mehr als $100 \mathrm{qm}$ ausgestattet sind, so ist die Situation in diesem Landkreis durchaus als problematisch zu bewerten. Hier sind 94 Prozent der Gemeinden ohne großflächigen Lebensmitteleinzelhandel und 65 Prozent der Bevölkerung haben keinen lokalen Zugang zu einer Verkaufsstelle mit mehr als $100 \mathrm{qm}$.

In diesem Landkreis sind die sozial-räumlichen Auswirkungen der Konzentration gut nachzuvollziehen. Es sind vor allem Disparitäten, die sich im ländlichen Raum zunehmend ausbilden. Während die Mittel- und Oberzentren gut versorgt sind, bricht die Nahversorgung im ländlichen Raum weg. Dabei ist der derzeit noch geringe Anteil von Discountern zu berücksichtigen. Wenn diese weiter expandieren, könnte es im Zuge eines Verdrängungswettbewerbes zu einem weiteren Rückzug der kleineren Supermärkte kommen.

\section{Die ältere Bevölkerung}

In denjenigen Gemeinden des Landkreises Daun, die vollkommen von lokalen Versorgungseinrichtungen ausgeschlossen sind, leben 4.527 Personen, die älter als 65 Jahre sind. Geht man davon aus, dass regelmäßige Einkäufe in größeren Verkaufstellen und Discountern nötig sind, um den Bedarf an Waren für den gesamten Haushalt zu decken, also ein gut ausgestatteter und großflächiger Lebensmitteleinzelhändler besucht werden muss, so sind es im Landkreis Daun 7.122 Personen, die über 65 sind und von einer "qualitativ hochwertigeren“ Nahversorgung ausgeschlossen sind. Das sind rund elf Prozent der Bevölkerung; in Bitburg-Prüm sind es 13 Prozent der Gesamtbevölkerung des Kreises.

Die wirtschaftliche Entwicklungsdynamik führt in Verbindung mit einer rückgängigen Bevölkerung im ländlichen Raum zu einer verstärkten Ausbildung von Disparitäten. In Ballungsgebieten können Verbraucher aufgrund intensiveren Wettbewerbs mit einer Verbreiterung des Angebots und mit Preissenkungen rechnen. In ländlichen Regionen und in städtischen Gebieten mit geringerer Siedlungsdichte sind hingegen Verschlechterungen für Verbraucher zu erwarten. Diese räumlichen Disparitäten werden durch soziale Disparitäten verschärft: mobile Bevölkerungsteile können die längeren Wege gut, das heißt überwiegend mit privaten Pkw, überbrücken. Weniger mobile Verbraucher sind dagegen in ihren Versorgungsund Auswahlmöglichkeiten zunehmend eingeschränkt. Die im Grundgesetz verankerte Leitvorstellung der „Gleichwertigkeit

\section{„Die Gleichwertigkeit der Lebensverhältnisse findet sich zunehmend in Frage gestellt."}

der Lebensverhältnisse" findet sich zunehmend infrage gestellt. Hieraus ergeben sich neue Herausforderungen für Ansätze der Marktgestaltung.

\section{Empfehlungen zur Nahversorgung}

- Förderung der Problemwahrnehmung: Der Rückgang der Nahversorgungsmöglichkeiten wird in der öffentlichen, politischen, aber auch wissenschaftlichen Diskussion kaum als Problem wahrgenommen. Das legen auch Anfragen des IÖW in mehr als 20 ländlichen Kreisen zum Thema Nahversorgung nahe. In den meisten Kreisen lagen weder Daten vor, noch wurde die lokale Versorgung als problematisch angesehen. In Kreisen mit Problembewusstsein war eine gewisse Resignation erkennbar, die sich aus dem Fehlen von Handlungsoptionen speiste. Daher sind vor allem die Problematisierung des Themas Nahversorgung und die Suche nach Lösungsmöglichkeiten auf allen gesellschaftlichen Ebenen geboten.

- Überkommunale planerische Ansätze ausbauen - Regionale Nahversorgungskonzepte entwickeln: Planerische Ansätze, wie sie derzeit im Bereich der Ansiedlungen von Versorgungszentren auf der "grünen Wiese“ installiert werden, bieten praktikable regionale Verfahrensweisen in Bezug auf die Einkaufsituation. Diese gilt es auch im Bereich der Nahversorgung verstärkt anzuwenden. Ziel muss eine verbesserte Kommunikation und Abstimmung zwischen den beteiligten Kommunen sein. Hier wäre erstens ein Monitoring anzustreben sowie zweitens die Erstellung eines gemeinsamen Wertekanons, in dem Zielvorgaben, Mindestausstattungen und so weiter gemeinsam festgelegt werden.

- Förderung des lokalen stationären Handels und Förderung von Mobilitätskonzepten: Zu vielen Bereichen der Grunddaseinsfunktionen wie Post, Telekommunikation, Energie oder ÖPNV sind in der Raumplanung Vorgaben festgehalten, die die Grundbedingungen für eine grundgesetzlich vorgeschriebene Gleichwertigkeit der Lebensverhältnisse in den Regionen herstellen. Solche Vorgaben existieren für die Nahversorgung nicht.

Dies gilt es möglicherweise zu ändern. So sind neben Nachbarschaftsläden auch andere neue Geschäftsmodelle vorstellbar. Zusätzlich sind gerade in den besonders dispers besiedelten Räumen Mobilitätskonzepte nötig, die sowohl die Mobilität der Verbraucher als auch die Mobilität der Waren im Blick haben. So gibt es mit dem mobilen Handel eine Angebotsform, die in unterversorgten Gebieten die Nahver- $\rightarrow$ 


\section{„Das Problem der Nahversorgung besteht nicht allein in Bezug auf Waren des täglichen Bedarfs."}

sorgung sicherstellen kann. Hier wäre die Suche nach neuen, flexiblen Geschäftsmodellen zu fördern. Beide Ansätze werden nicht ohne zusätzliche Finanzierungsinstrumente auskommen.

I Gesellschaftliche Verantwortung des Einzelhandels: Die Unternehmen des Lebensmitteleinzelhandels und vor allem die größten fünf Unternehmen ziehen sich weiter aus der Fläche zurück und reduzieren ihre kleinformatigen Angebote. Zwar werden auch neue Formate entwickelt, die auf kleine Einzugsgebiete orientiert sind. Diese können sich bislang aber nur in begrenztem Umfang durchsetzen. Auch die "Großen“ der Branche sollten ihrer gesellschaftlichen Verantwortung, einen Beitrag zur Sicherung der Nahversorgung zu leisten, nachkommen. Mit der offensiven Einrichtung von kleinen Ladenformaten in schlecht versorgten Regionen oder mit der Unterstützung von bürgerschaftlichen Initiativen wie Nachbarschaftsläden sollte auch ohne politische Regulation ein sichtbarer Beitrag zu leisten sein.

Abschließend ist daran zu erinnern, dass das Problem der Nahversorgung nicht allein in Bezug auf Waren des täglichen Bedarfs besteht. Ähnliche Schwierigkeiten sind hinsichtlich einer Reihe von wirtschaftlichen Aktivitäten festzustellen. Insofern sollte geprüft werden, inwieweit eine Koordination zwischen den unterschiedlichen Problemgebieten möglich ist. Dies sollte nicht zuletzt aufgrund der zunehmenden Problemlagen geschehen, die als Folge der Alterung der Gesellschaft auf die ländlichen Räume zukommen. Es setzt hier ein für viele Bevölkerungsgruppen nachteiliger Prozess ein, den umzugestalten es aller gesellschaftlicher Akteure bedarf.

\section{Literatur}

Bundesverband des Deutschen Lebensmitteleinzelhandels (BVL) (Hrsg.): Struktur und Leistungszahlen des Lebensmittel-Einzelhandels 2003 nach Einzelhandelstypen. Berlin 2004. Download unter: www.lebensmittelhandel-bvl.de

Bundesamt für Bauwesen und Raumordnung (Hrsg.): INKAR. CD-Rom, Bonn 2003.

EHI - EuroHandels-Institut (Hrsg.): Handel aktuell 2004. Strukturkennzahlen und Profile des deutschen und internationalen Handel. Ohne Ortsangabe 2004

Federsel-Lieb, C.: Kommunikationspolitik im Seniorenmarkt. Bayreuth 1992. IKB (Hrsg.): Einzelhandel vor massivem Strukturwandel - Perspektive 2020 Ohne Ortsangabe 2003

M + M Eurodata (Hrsg.): Pressemitteilung November 2000.

\section{Anmerkung}

(1) Die Top fünf sind die Metro-Gruppe, Rewe-Gruppe, Edeka/AVA-Gruppe und Aldi.

I AUTOREN + KONTAKT

Christian Kuhlicke ist Doktorand am Department Stadt- und Umweltsoziologie, Umweltforschungszentrum Leipzig-Halle (UFZ)

Ulrich Petschow ist Leiter des Forschungsfeldes Umweltökonomie und -politik am Institut für ökologische Wirtschaftsforschung (IÖW).

UFZ, Permoserstraße 15, 04318 Leipzig. E-Mail: christian.kuhlicke@ufz.de, IÖW, Potsdamerstr. 105, 10875 Berlin. E-Mail: ulrich.petschow@ioew.de
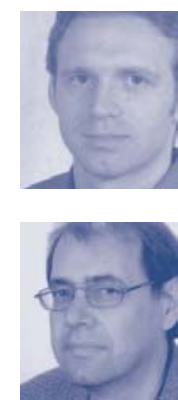
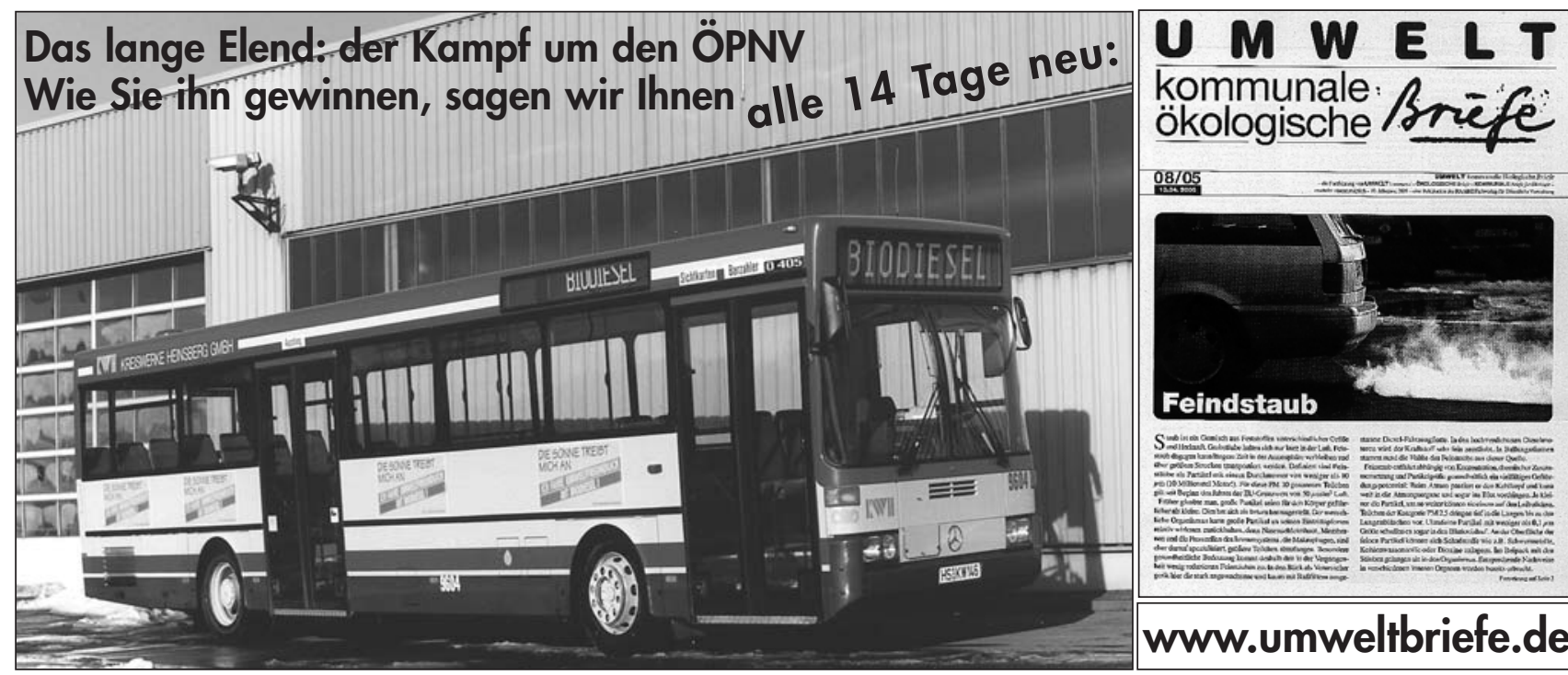

\section{5}
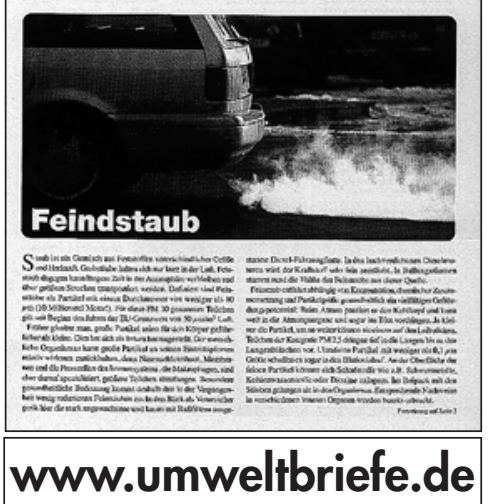
(c) 20I0 Authors; licensee IÖW and oekom verlag. This is an article distributed under the terms of the Creative Commons Attribution Non-Commercial No Derivates License (http://creativecommons.org/licenses/by-nc-nd/3.o/), which permits unrestricted use, distribution, and reproduction in any medium, provided the original work is properly cited. 